\title{
Quistes cartilaginosos del pubis: diagnóstico radiológico
}

\section{Cartilaginous pubic cyst: radiological diagnosis}

\author{
Jaime Iglesias-Gordo*, Agustín E. Borja-Consigliere, Francisco J. Borja-Consigliere y Cristina González-Sainza \\ Servicio de Radiología, Osatek Resonancia Magnética, Hospital Universitario Donostia, Donostia-San Sebastián, Guipúzcoa, España
}

\begin{abstract}
Resumen
Los quistes cartilaginosos del pubis son lesiones muy raras cuya forma de presentación típica es la aparición de una masa de consistencia muy dura en la región perineal anterior, pudiendo simular procesos malignos, con o sin sintomatología asociada, fundamentalmente dolorosa o urológica. Se trata de un proceso benigno, de probable naturaleza degenerativa, que suele producirse en mujeres de edad media o avanzada, a menudo con antecedente de varios partos. Tienen características radiológicas típicas que permiten un diagnóstico radiológico preciso en la mayoría de los casos, principalmente mediante resonancia magnética. Con la presentación de un caso y la revisión de la literatura analizamos las principales características clínicas y el diagnóstico por imagen de esta patología, mencionando las opciones para su manejo.
\end{abstract}

Palabras clave: Quiste cartilaginoso. Sínfisis del pubis. Radiología. Diagnóstico.

\section{Abstract}

Cartilaginous pubic cysts are extremely rare lesions whose typical form of presentation is the appearance of a mass of very hard consistency in the anterior perineal region that can simulate a malignant process, with or without pain or urological associated symptoms. They are benign in nature with a probable degenerative origin that usually occurs in middle-aged or elderly women, frequently with a history of multiparity. They have typical radiological features that can allow an accurate diagnosis in most cases, mainly by magnetic resonance imaging. Through this case and review of the literature, we analyze the main clinical aspects and diagnostic imaging of this pathology, mentioning the options for its management.

Key words: Cartilaginous cyst. Pubic symphysis. Radiology. Diagnosis.

\section{Introducción}

Los quistes cartilaginosos del pubis son lesiones muy raras, descritas principalmente en mujeres multíparas de edad avanzada. Suelen presentarse como masas en la región perineal anterior, de consistencia pétrea, que pueden simular tumores agresivos, aunque en realidad se trata de una patología benigna. Su diagnóstico puede ser difícil debido a la poca casuística y la variedad de su presentación clínica, aunque utilizando una correcta evaluación radiológica, en especial mediante resonancia magnética, se puede realizar un diagnóstico preciso en la mayor parte de las ocasiones sin necesidad de recurrir a procedimientos invasivos. En esta comunicación presentamos esta patología a través del diagnóstico de un caso, revisando sus características clínicas y radiológicas.
Correspondencia:

*Jaime Iglesias Gordo

E-mail: jdiglesias@osatek.eus

0717-9308 / @ 2021 Sociedad Chilena de Radiología. Publicado por Permanyer. Éste es un artículo open access bajo la licencia CC BY-NC-ND
Fecha de recepción: 08-02-2021

Fecha de aceptación: 23-09-2021
Disponible en internet: 03-12-2021

Rev Chil Radiol. 2021;27(4):177-180

www.resochradi.com (https://creativecommons.org/licenses/by-nc-nd/4.0/). 


\section{Presentación del caso}

Mujer de 60 años derivada desde la consulta de ginecología con los antecedentes clínicos de aparición de una tumoración indolora en la región vulvar de consistencia muy dura, la cual, en la ecografía perineal realizada en ese servicio, mostró ecogenicidad mixta, por lo que se solicitó estudio complementario con resonancia magnética (RM). No se disponía de estudios mediante otras técnicas de imagen en el sistema de archivo y comunicación de imágenes. En la historia clínica se halló el antecedente de tres gestaciones con parto, sin patologías personales relevantes.

En la RM (Fig. 1) se observó una lesión redondeada adyacente a la sínfisis del pubis, situada en la línea media, en íntima relación con el cartílago de la sínfisis, la cual presentaba extensión principalmente infrapúbica y, en menor medida, suprapúbica. Era una lesión circunscrita, hipointensa en T1, hiperintensa de forma heterogénea en T2 y STIR, con fino realce lineal periférico y sin realce central tras la administración de contraste. No se apreciaban invasión de estructuras vecinas ni lesiones sospechosas en el hueso adyacente de la pelvis ni en la región genital. Se diagnosticó como quiste cartilaginoso subpúbico y se acordó el seguimiento por los servicios de ginecología y traumatología, ya que se trababa de una lesión asintomática. Posteriormente se solicitaron una radiografía simple (Fig. 2A) y otra RM a los 4 meses (Fig. 3), que demostró la estabilidad de la lesión. Más tarde, por otro motivo clínico, se solicitó una tomografía computarizada (TC) (Fig. 2B y C). Hasta la fecha, con más de 2 años de evolución, la paciente permanece asintomática y la lesión se encuentra estable en los sucesivos controles.

\section{Discusión}

A pesar de su rareza y de la posible alarma generada por su forma de presentación, los quistes cartilaginosos del pubis son afecciones benignas con unas características radiológicas típicas que pueden permitir un diagnóstico certero en la mayoría de las ocasiones, evitando así procedimientos invasivos en una región anatómica difícil y las posibles complicaciones asociadas a la realización de estos.

Fueron descritos por primera vez en 1996 por Alguacil-García y Littman ${ }^{1}$, quienes publicaron dos casos de lesiones inusuales que ocasionaban problemas en los genitales inferiores en mujeres de edad avanzada. En ambos casos se trataba de lesiones

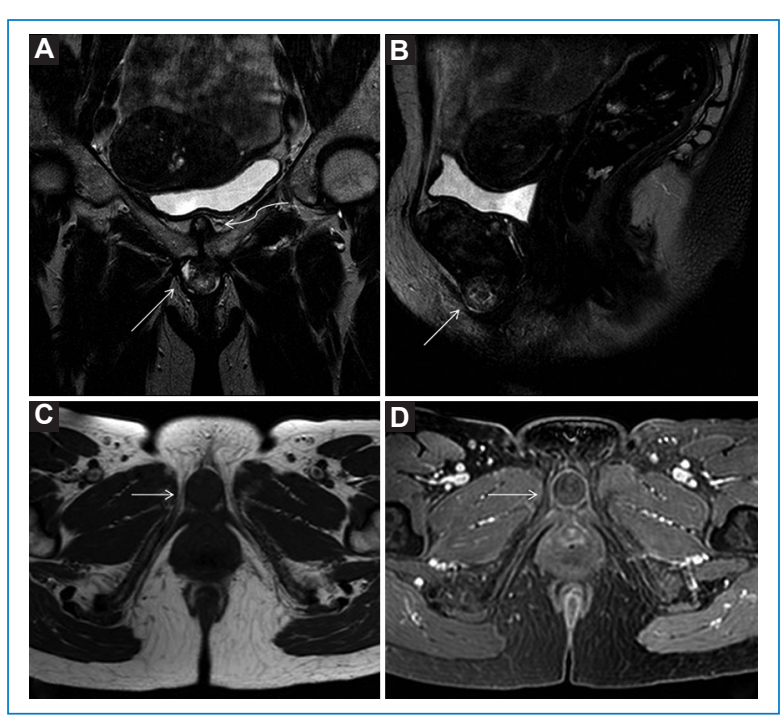

Figura 1. A: resonancia magnética en secuencias coronal y B: sagital potenciadas en T2, C: axiales T1 y T1 eco de gradiente con supresión de la grasa y $\mathbf{D}$ : con administración de gadolinio intravenoso. Se observa una lesión redondeada en el margen inferior de la sínfisis del pubis (flechas rectas), aunque también con extensión desde su margen superior (flecha curva). A y B: lesión hiperintensa de forma heterogénea en T2. C y D: lesión hipointensa en las secuencias potenciadas en T1. D: fino realce periférico tras la administración de contraste. Los huesos del pubis tienen una intensidad de señal normal y no se observan realce interno, invasión de estructuras vecinas ni otros hallazgos que sugieran agresividad. Se diagnosticó como quiste cartilaginoso del pubis.

quísticas recubiertas por una cápsula fibrosa de colágeno que contenía tejido cartilaginoso degenerado, sugiriendo su origen en la sínfisis del pubis por su localización y similitud con los cambios degenerativos constatados en el análisis anatomopatológico de esta articulación. El primer caso descrito desde el punto de vista radiológico se publicó en $2004^{2}$ y en él se mostraron las características mediante RM de una lesión compatible con lo descrito previamente por AlguacilGarcía y Littman ${ }^{1}$ en una mujer de 70 años. Hasta ahora hay muy pocos casos publicados, lo cual dificulta su conocimiento y diagnóstico.

Se trata de una lesión de etiología desconocida que fundamentalmente se ha descrito en mujeres multíparas de edad avanzada, lo que lleva a proponer una probable relación con cambios degenerativos en la sínfisis del pubis que progresan con posibles traumatismos 0 en el parto ${ }^{3}$, aunque también hay algunos casos descritos en hombres. La presentación clínica 


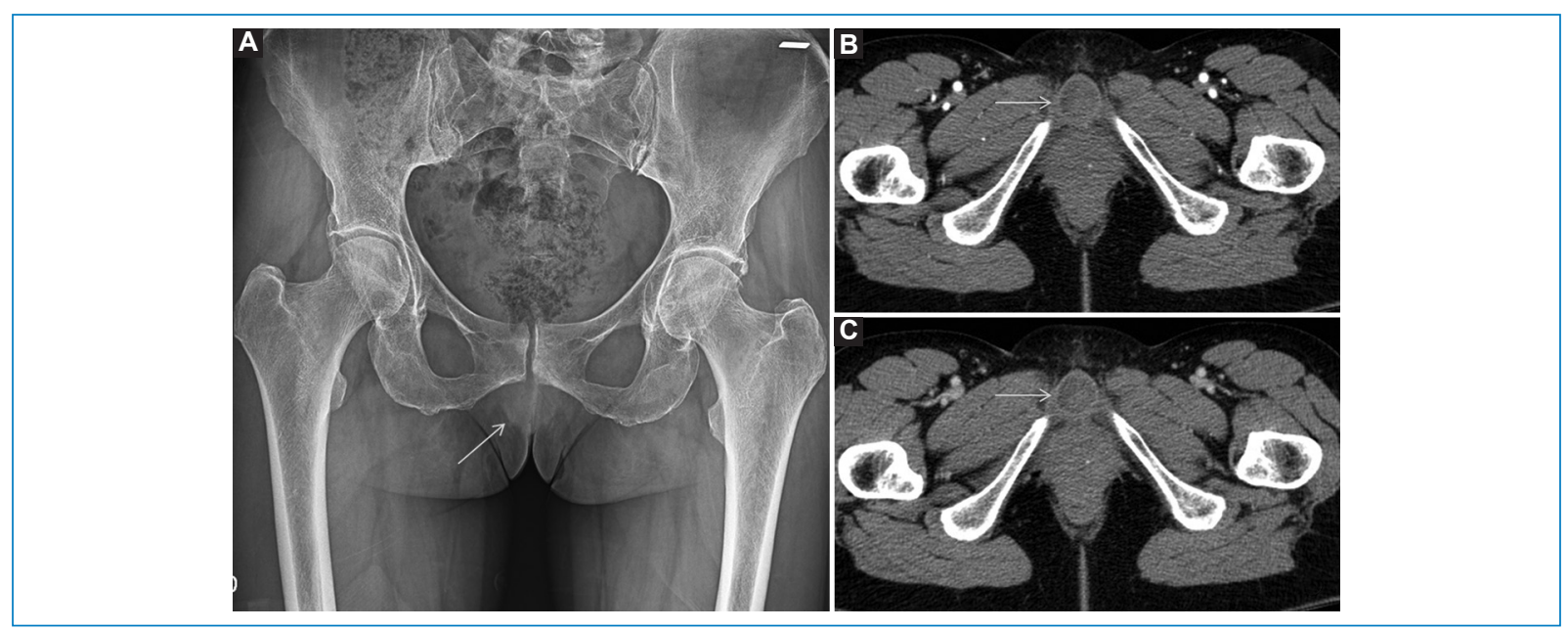

Figura 2. A: radiografía simple de pelvis en la que se aprecian ligeros cambios degenerativos en la sínfisis del pubis y una estructura ovalada (flecha) con densidad de partes blandas que se extiende principalmente desde el margen inferior de la articulación, aunque también en menor medida desde su margen superior. B y C: tomografía computarizada axial con contraste en fases arterial y portal realizada posteriormente por otro motivo clínico, en la que se aprecia la lesión circunscrita de baja atenuación con fino realce periférico (flechas), sin realce interno ni otros datos de agresividad.

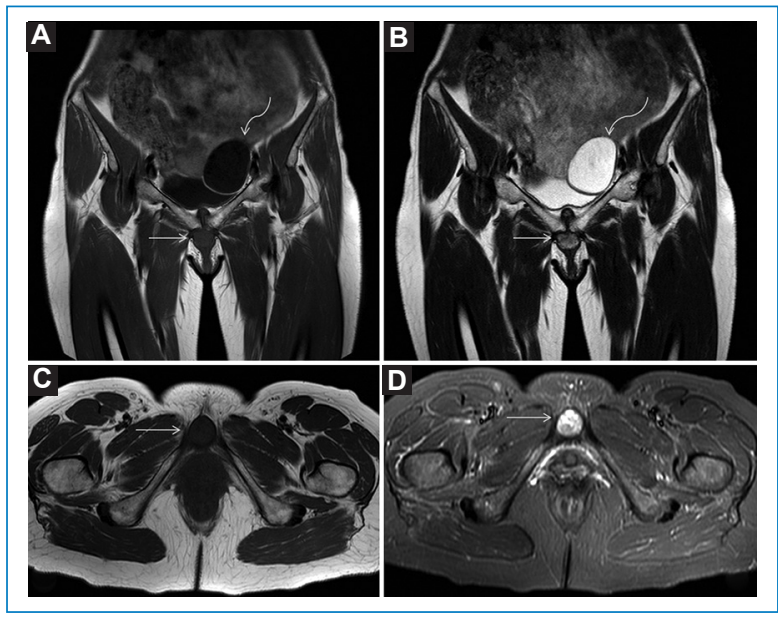

Figura 3. Resonancia magnética (RM) en secuencias coronales $\mathrm{T} 1$ y $\mathrm{T} 2$ y axiales T1 y STIR, en las que se observa la estabilidad de la lesión (flechas rectas). Como hallazgo incidental se identifica una lesión quística anexial izquierda (flechas curvas en A y B: que ya estaba presente en la RM inicial, aunque por el movimiento y el plano de corte no se visualiza en las imágenes mostradas, y también se encontraba estable en los controles ginecológicos.

puede ser muy variada, con aparición de una masa en la región perineal anterior que puede ser indolora 0 bien asociar molestias en la región vulvar o suprapúbica, con otra posible sintomatología como disuria, dispareunia, retención urinaria, infecciones recurrentes del tracto urinario ${ }^{4} 0$ incluso disfunción eréctil en un caso de un varón ${ }^{5}$.

En el diagnóstico radiológico es fundamental demostrar la asociación de la lesión con la sínfisis del pubis y su localización en la línea media, en general en su margen inferior, aunque también excepcionalmente puede tener extensión posterior o superior, como se demuestra en este caso, que lo convierte en aún más singular y podría llevar a replantear su nombre en la literatura médica como «quiste cartilaginoso del pubis» en lugar de «subpúbico», como mayoritariamente se ha ido recogiendo hasta ahora. Su aspecto puede variar en función de su contenido en mucina y elementos condroides. En la radiografía simple puede aparecer como una lesión con densidad de partes blandas (Fig. 2A) o incluso pasar desapercibida, normalmente asociada a cambios degenerativos en la sínfisis del pubis con irregularidades marginales y esclerosis. En la ecografía suele presentarse como una lesión muy dura de ecogenicidad mixta debido a su contenido ${ }^{6}$, solo anecoica en las zonas de necrosis y degeneración quística. Mediante TC se identifica una lesión redondeada de baja atenuación relacionada anatómicamente con la sínfisis del pubis ${ }^{7}$ (Fig. 2B y C), aunque la prueba de imagen de mayor precisión es la RM, en la que además de demostrar esta morfología y su relación anatómica se pueden observar características típicas en su intensidad de señal, siendo hipointensa en T1, hiperintensa de forma heterogénea en T2, normalmente con 
leve realce periférico tras la administración de contraste y sin realce interno ni alteración de la señal del hueso adyacente (Figs. 1 y 3 ).

El diagnóstico diferencial se realiza con lesiones quísticas en la zona perineal ${ }^{8}$, como divertículos uretrales o quistes de las glándulas de Skene, de Bartolino o de Gartner, fáciles de diferenciar por su localización y por la ausencia de contenido, salvo complicación. Además, se deben descartar abscesos y lesiones o neoplasias procedentes del clítoris, la uretra o la vagina, así como de elementos óseos o cartilaginosos adyacentes, como condrosarcomas u otro tipo de sarcomas, sin olvidar la posibilidad de metástasis. Estas patologías, a diferencia de los quistes cartilaginosos del pubis, no presentan una estrecha relación con la sínfisis del pubis ni sus características típicas por imagen. Utilizando esta descripción podremos alcanzar un alto grado de seguridad diagnóstica y proponer controles en los casos en que la situación clínica lo permita, evitando así procedimientos invasivos innecesarios.

No hay una opción estandarizada de tratamiento debido a los pocos casos publicados. La mayoría de los recogidos en la literatura optan por la observación en los pacientes asintomáticos, sin haber detectado recurrencias hasta la fecha y con solo una regresión espontánea ${ }^{9}$, reservando el tratamiento quirúrgico para los casos sintomáticos ${ }^{10}$.

\section{Financiamiento}

Todos los autores declaramos que no se ha producido ningún tipo de financiamiento para la realización de este artículo.

\section{Conflicto de intereses}

Todos los autores declaramos que no tenemos conflicto de intereses.

\section{Responsabilidades éticas}

Protección de personas y animales: Los autores declaramos que para esta investigación no se han realizado experimentos en seres humanos ni en animales.

Confidencialidad de los datos: Los autores declaramos que hemos seguido los protocolos de su centro de trabajo sobre la publicación de datos de pacientes.

Derecho a la privacidad y consentimiento informado: Los autores hemos obtenido el consentimiento informado de los pacientes y/o sujetos referidos en el artículo. Este documento obra en poder del autor de correspondencia.

\section{Bibliografía}

1. Alguacil-Garcia A, Littman C. Subpubic cartilaginous cyst: report of two cases. Am J Surg Pathol. 1996;20:975-9.

2. Kim CE, Beasley HS. MRI diagnosis of subpubic cartilaginous cyst. Am J Roentgenol. 2004;182:144-6.

3. Elmelund M, Thind P, Klarskov N. Retropubic cartilaginous cyst presenting as stress urinary incontinence. Int Urogynecol J. 2014;26:455-7.

4. Price G, McNicholas T, Buckingham S, Chang S. Subpubic cartilaginous cyst: an unusual cause of urinary tract infection. JRSM Open. 2018;9:1-3.

5. Faraj K, Gross K, Beauchamp C, Castle E, Khan A. Surgical excision of an acutely symptomatic subpubic cartilaginous cyst in a 70 year old male. Urol Case Rep. 2018;20:62-4.

6. Lee HN, Ahn SE, Park JS, Park SY, Jin W, Ryu KN. Sonographic appearance of a cartilaginous cyst from the symphysis pubis mimicking a mass in the urinary bladder. J Clin Ultrasound. 2014;42:562-4.

7. Ergun $\mathrm{T}$, Lakadamyali $\mathrm{H}$, Aydin $\mathrm{O}$. Subpubic cartilaginous cyst: incidental finding detected by abdominopelvic computed tomography. Radiat Med. 2008;26:46-9.

8. Viester MDM, Schotman M. Three female patients with a periurethral mass: from various complaints to rare pathology. BMJ Case Rep. 2020;13:1-4.

9. Nishisho T, Takao S, Miyagi R, Toki S, Nagamachi A, Sairyo K. Complete spontaneous regression of a subpubic cartilaginous cyst: a case report. J Med Investig. 2016;63:319-22.

10. Taniguchi $Y$, Kamada $H$, Sugaya $H$, Nishino $T$, Mishima $H$, Ochiai $N$, et al. Subacute urinary retention due to a subpubic cartilaginous cyst treated with surgical resection and internal fixation: a case report and review of the literature. Case Rep Orthop. 2018;2018:1-7. 